\title{
Genetic Variability, Diversity and Character Association in Sponge Gourd [Luffacylindrica (Roem.) L.]
}

\author{
J. Suresh Kumar ${ }^{1}$, M.K. Pandit ${ }^{2}$ and T. Lakshmi Pathy ${ }^{3}$ \\ ${ }^{1}$ ICAR-Central Tuber Crops Research Institute, Thiruvananthapuram, Kerala, -695017, India \\ ${ }^{2}$ Department of Vegetable Crops, Faculty of Horticulture, BCKV, West Bengal, India \\ ${ }^{3}$ Division of Crop Improvement, ICAR - Sugarcane Breeding Institute, Tamil Nadu, India \\ *Corresponding author
}

\begin{abstract}
A B S T R A C T
The present study was conducted to determine phenotypic performance, genetic variability, heritability, genetic advance, diversity, correlation and path analysis for yield and seventeen yield attributing characters of forty five sponge gourd germplasm. PCV was higher than GCV for all the traits studied. High PCV and GCV were recorded for number of primary branches, number of fruiting nodes on main stem, sex ratio, fruits per plant and yield per plant. Thirteen characters showed high heritability coupled with high genetic advance. Correlation coefficient study indicated that yield per plant had highly significant positive relationship with number of primary branches, fruiting nodes on main stem, fruit length, fruit weight, fruits per plant, seeds per fruit and 100 seed weight. Path co-efficient analysis showed that fruits per plant (0.7013) and number of seeds per fruit $(0.6833)$ exhibited the highest positive direct effect on yield per plant. Based on Mahalanobis' $\mathrm{D}^{2}$ statistics, the forty five genotypes were grouped into seven different clusters. Maximum intra cluster distance was in cluster- III (541.13) where maximum inter-cluster distance was between cluster- IV and cluster- V (5177.9). Characters namely yield per plant, fruit weight and number of fruiting nodes on main stem showed maximum contribution towards divergence, hence considering these characters in selection of diverse parents during hybridization program manifests high heterosis.
\end{abstract}

\section{Introduction}

Sponge gourd or smooth gourd or dishcloth gourd or smooth loofah or vegetable sponge [Luffa cylindrica (Roem.) L.] $(2 \mathrm{n}=2 \mathrm{x}=26)$ is one of the minor cucurbitaceous vegetable crop with old world origin in subtropical Asian region including particularly India (Kalloo, 1993; Swarup, 2006). This crop has been cultivating for centuries in the Middle
East, India, China, Japan and Malaysia. In India, it is cultivated on both commercial scale and in kitchen gardens during the spring summer and rainy season. Luffa has nine species out of which Luffa acutangula (L.) Roxb., L. cylindrica M. Roem., L. echinta Roxb., L. graveolens, L. tuberose Roxb., $L$. umbellata are found in India. Luffa acutangula (ridge gourd) and Luffa cylindrica (sponge gourd) are grown throughout India in tropical 
and subtropical climate. Luffa acutangula has three varieties: var. acutangula is grown in South East Asia and other tropical areas; var. amara, a wild form is confined to peninsular India, while var. forskallii (Harms.) Heiser and Schilling, another wild form is confined to Yemen. Luffa echinata grows in natural habitat in western Himalayas, central India and Gangetic plains. Luffa graveolens is a wild species distributed in parts of North Central India

The tender or immature fruits are cooked as vegetable, used in the preparation of chutneys and curries and tender fruits are easily digestible and increase appetite when consumed. Sponge gourd is a highly nutritive vegetable and contains moisture of $93.2 \mathrm{~g}$, protein $1.2 \mathrm{~g}$, fat $0.2 \mathrm{~g}$, carbohydrates $2.9 \mathrm{~g}$, vitamins (thiamine $0.02 \mathrm{mg}$, riboflavin $0.06 \mathrm{mg}$, niacin $0.4 \mathrm{mg}$ and $\beta$ carotene 120 $\mathrm{mg}$ ), minerals (calcium $36 \mathrm{mg}$, phosphorous $19 \mathrm{mg}$ and iron $1.1 \mathrm{mg}$ ) and fibers $0.20 \mathrm{~g}$ per $100 \mathrm{~g}$ of edible portion (Gopalan et al., 1999). Sponge gourd fruits contain more protein and carotene than ridge gourd (More and Shinde, 2001). Sponge gourd is a minor crop and its cultivation is not yet flourished at commercial scale and exact area of cultivation is not known.

Sponge gourd is an annual climber and monoecious vegetable but different sex forms like hermaphrodite, staminate and pistillate etc. are commonly found in nature (Takahashi, 1980) flowers are large yellow in colour, Group of male and single female flowers are formed in leaf axil. The existence of different size of fruits ranging from a few centimetres to one meter, different fruit shapes and colours (light green, green and dark green with light white stripes, etc.) indicates the presence of wide genetic variability. To develop a new variety there is need of high magnitude of genetic variability in the base material and the vast of variability for desired characters, variation may exist or created, is the first step to any crop improvement programme (Singh, 2000). To know the extent of variability present in a population, evaluation of large number of germplasm lines is the first line of work. This improvement in any crop is based on the extent of genetic variation and magnitude of available beneficial genetic variability. Some of the biometric parameters include genotypic (GCV) and phenotypic (PCV) coefficients of variation. High value of these coefficients indicates wider diversity. Similarly, narrow difference between GCV and PCV reveals low sensitivity to the environmental effects. Another indicator of variability is heritability, which is the ratio of genetic variance to total variance. This is broad sense heritability and gives an idea about that portion of observed variability which is attributable to genetic differences. Heritability estimates supplemented by genetic variance are more meaningful. Heritability is a component in the computation of expected progress which is most meaningful when accompanied by genetic advance. Genetic advance would be more in cases where the additive genetic variance is more than nonadditive genetic variance (Lush, 1949).

Crop improvement through successful selection programme is only achieved using valid information about the correlation and genetic variability of traits of interest. Correlation studies among yield and other traits of the crop will be of interest to breeders in planning hybridization programmes and evaluating the individual plants in the segregating populations. Knowledge of genetic diversity among existing cultivars of any crop is essential for long term success in breeding programme and to maximize the exploitation of the germplasm resources (Rabbani et al., 2012). The genetically diverse parents are always able to produce high heterotic effects and great frequency of desirable segregants in further generations. $\mathrm{D}^{2}$ 
statistic is a useful tool to measure genetic divergence among genotypes in any crop as developed by Mahalanobis (1936). However, in the present study, an attempt has been made together information on genetic variability, heritability, genetic gain, correlation and path analysis for different characteristics of sponge gourd, so as to select the potential parents for breeding programme to attain the anticipated improvement in fruit yield per plant.

\section{Materials and Methods}

\section{Location and experiment}

The study was conducted in Alluvial zone of West Bengal at Horticultural Research Station, Mandouri, Bidhan Chandra KrishiViswavidyala, West Bengal, India during March 2014 and July 2015.

\section{Plant material and source}

Total forty five genotypes were collected from ICAR-NBPGR, New Delhi; ICAR-IIVR, Varanasi; parts of West Bengal, Andhra Pradesh and Bihar states of India. It was evaluated for two years during March 2014 and March 2015. The seeds were sown in pits taken at a row spacing of $1.0 \mathrm{~m}$ and intra row spacing of $0.75 \mathrm{~m}$ in randomized block design (RBD). Well decomposed FYM (Farm Yard Manure) was incorporated into the soil @ 10 tonnes per hectare.

The experimental plots were fertilized @ 40 $\mathrm{kg} \mathrm{N}, 20 \mathrm{~kg} \mathrm{P}_{2} \mathrm{O}_{5}$ and $20 \mathrm{~kg} \mathrm{~K}_{2} \mathrm{O}$ per hectare. Half of the nitrogen, full dose of phosphorus and potash were applied as basal. The remaining half of the nitrogen was applied in two equal splits at an interval of one month starting from sowing. The crop was grown under irrigated conditions duly adopting recommended cultural practices (Singh and Singh, 2009). Need based plant protection measures were also taken up to control the pests and diseases.

\section{Observations}

The observations were recorded in five randomly selected plants from each replication for vine length, number of primary branches, number of fruiting nodes on main stem, days to first staminate flower appearance, days to first pistillate flower appearance, days to $50 \%$ flowering, span of flowering, sex ratio, node at which $1^{\text {st }}$ female flower appearance, days to harvestable maturity from anthesis, fruit length, diameter of the fruit, fruit weight, number of fruits per plant, number of seeds per fruit, seed index (100 seed weight) and fruit yield per plant.

\section{Statistical analysis}

The data recorded were subjected to genotypic coefficient of variation (GCV), phenotypic coefficient of variation (PCV), broad sense heritability $\left(\mathrm{H}^{2}\right)$, genetic advance as per cent of mean (GAM), correlation (genotypic and phenotypic) and path coefficients were computed by the methods suggested by Panse and Sukhatme (1985). Analysis of genetic divergence was done according to Mahalanobis' $\mathrm{D}^{2}$ (1936) statistics. The analysis was computed by using computer software program Windostat version 9.3 from Indostat services, Hyderabad, India.

\section{Results and Discussion}

The analysis of variance showed highly significant differences among 45 genotypes for all the characters indicating differences among the genotypes under study. The extent of variability in sponge gourd genotypes were measured in terms of mean, range, PCV, GCV, heritability and genetic advance (Table 1). Range of all traits revealed that there was a wide variation among the collected genotypes. The range of variation was widest for fruit weight ( 74.96 to $184.29 \mathrm{~g}$ ) followed by fruit length (11.37 to $25.54 \mathrm{~cm})$. The narrowest 
range was observed in seed index (10.73 to 13.01). PCV were higher than their corresponding GCV, which is obvious due to environmental influence. Relatively higher magnitude (>20.01\%) of PCV and GCV were recorded in number of primary branches (24.56\%, 24.94\%), number of fruiting nodes on main stem $(34.46 \%, 34.67 \%)$, sex ratio $(22.29 \%, 24.35 \%)$, fruits per plant $(22.97 \%$, $23.61 \%)$ and yield per plant $(44.16 \%$, 46.66\%). This implies that maximum variability is existed in the genotypes for aforesaid traits and there is good scope for improvement of these characters. Similar results were also reported byGowda et al., (2011), Rabbani et al., (2012), Dubey et al., (2013), Choudhary et al., (2014), Koppad et al., (2015), Ananthan and Krishnamoorthy (2017), Karthik et al., (2017) in ridge gourd, Khule et al., (2011), Kumar et al., (2013), Sharma et al., (2017) in sponge gourd. The differences between values of PCV and GCV were less for all traits except number of seeds per fruit $(16.31 \%, 20.64 \%)$. This suggests that the traits were less influenced by environment and hence they could be improved by following simple phenotypic selection. Similar results were also reported by Gowda et al., (2011), Rabbani et al., (2012), Choudhary et al., (2014) and Koppad et al., (2015) in ridge gourd.

GCV can further be investigated with the help of heritability estimates. While co-efficient of variation measure the magnitude of variability present in a population, heritability indicates the reliability with which the genotype is recognized by its phenotypic expression. High heritability estimates provides a clue that the characters would exhibit high response to selection. High heritability( $>60 \%$, Robinson 1966) and genetic advance as per cent of mean (>20\%, Johnson et al., 1955) estimates were recorded for vine length $(89.55,22.70)$, number of primary branches $(96.99,49.83)$, number of fruiting nodes on main stem (98.79,
$70.55)$, sex ratio $(83.81,42.04)$, fruits per plant $(94.58,46.01)$, yield per plant (89.55, 86.08 ), days to pistillate flower appearance $(87.58,21.45)$, days to $50 \%$ flowering $(85.29$, 22.85), node at first female flower appearance (91.44, 36.89), fruit length $(81.86,35.26)$, fruit diameter $(83.06,21.31)$, fruit weight $(74.15$, $25.19)$ and number of seeds per fruit (62.41, 26.54) respectively. This indicated that all the above said traits were under the influence of additive gene action and simple selection based on phenotypic performance of these traits would be effective. Similar findings were reported by Choudhary et al., (2014), Ananthan and Krishnamoorthy (2017) in ridge gourd, Khule et al., (2011), Kumar et al., (2013), Sharama et al., (2017) in sponge gourd.

Correlation studies showed that for most character pairs, genotypic and phenotypic associations were in the same direction and genotypic estimates were higher than the phenotypic ones, indicating an inherent association between the characters (Table 2). The correlation studies revealed that yield per plant had positive and highly significant correlation with number of primary branches $(0.4689,0.4747)$, fruiting nodes on main stem $(0.6141, \quad 0.6168)$, fruit length $(0.3309$, $0.3396)$, fruit weight $(0.4677,0.4475)$, fruits per plant $(0.6607,0.6648)$, seeds per fruit $(0.5401,0.4772)$ and 100 seed weight $(0.2462$, 0.1978). These characters were most important selection criteria as they showed significant positive correlation and positive direct effect with yield. Days taken for first staminate flower appearance, days taken for first pistillate flower appearance, days to $50 \%$ flowering, sex ratio, node at which first female flower appearance and days to harvestable maturity from anthesis were found to be negatively associated with total yield per vine but in desirable direction because negative values of these traits are beneficial and contribute positively to the yield per vine. This 
suggested that direct selection based on these traits would be rewarding for yield improvement. Such results were earlier reported by Pandey et al., (2012), Kumar et al., (2013), Dubey et al., (2013), Yadav et al., (2017) in sponge gourd. Rabbani et al., (2012), Gowda et al., (2012), Dubey et al., (2013), Narasannavar et al., (2014), Choudhary et al., (2014) and Koppad et al., (2015), Varalaxmi et al., (2015), Ananthan and Krishnamoorthy (2017) in ridge gourd.

Path analysis helps in understanding the magnitude of direct and indirect contribution of each character on the dependent character, viz., yield. The results presented in Table 3, revealed that's different component traits viz., fruits per plant (0.7013), Number of seeds per fruit (0.6833) and seed index (0.2293) exhibited positive direct effects towards yield per vine along with significant positive correlation with yield, indicating the importance of these characters in direct selection for yield. Similar results were reported by Pandey et al., (2012), Kumar et al., (2013), Yadav et al., (2017), Sharma et al., (2017) in sponge gourd, Rabbani et al., (2012), Singh et al., (2012), Dubey et al., (2013), Narsannavar et al., (2014), Choudhary et al., (2014), Koppad et al., (2015), Varalaxmi et al., (2015), Ananthan and Krishnamoorthy (2017) in ridge gourd.

All the forty five genotypes were grouped into 7 different non-overlapping clusters following Mahalanobis's methods (Table 4). Out of the seven clusters, cluster- I was largest comprising 18 genotypes, followed by clusterII comprising 12 genotypes, cluster -III with 9 genotypes, cluster -IV with 3 genotypes. Three clusters were single genotype clusters, these clusters were V, VI and VII. The inter cluster distances were higher than intra cluster distances (Table 5). Maximum intra cluster $\mathrm{D}^{2}$ distance was found to be in clusterIV(541.13) followed by cluster-III (519.62) and cluster- II (351.53). Maximum inter cluster distance was found to be between cluster- IV and cluster- V (5177.9) followed by cluster- IV and cluster- VI (4034.09) and cluster- IV and cluster- VII (3552.24). genotypes belonging to distant clusters may be used as parents in hybridization programme for exploiting high heterosis in $\mathrm{F}_{1}$ generation as reported by Choudhary et al., (2011), Rabbani et al., (2012), Yadav et al.,(2016), Quamruzzaman et al., (2011), Khule et al., (2012), Singh et al., (2008).

The comparison of clusters means revealed considerable differences among the clusters of different quantitative characters (Table 6). The cluster mean for vine length was highest in cluster- I $(3.45 \mathrm{~m})$ followed by cluster $-\mathrm{II}$ and IV $(3.25 \mathrm{~m})$ and lowest vine length for the cluster-VII $(2.74 \mathrm{~m})$. For number of primary branches cluster- II (3.89) had highest value followed by clusters- VI, VII (3.60) and lowest value for cluster-I (2.66). Number of fruiting nodes on main stem highest value was recorded by cluster- II (5.92) and lowest by cluster- V (3.20). For days to first staminate flowers appearance cluster-VII (47.96) has taken more number of days while cluster- IV (37.95 days) recorded minimum number of days. Maximum number of days to first pistillate flower appearance was observed in cluster-VII (52.63 days), while minimum number of days was observed in cluster- IV (41.71 days). Cluster- IV (45.08) recorded minimum days to $50 \%$ flowering and maximum for the cluster- VII (57.50). Cluster mean value for span of flowering ranged from 36.49 to 38.43 , highest being for the cluster-III and lowest in the cluster VII. Sex ratio on whole plant ranged from 16.09 to 24.67 ; the highest is being for the cluster- I and the lowest for the cluster VI. Cluster -IV had recorded lowest (10.72) for the node at which the first female flower appeared, while clusterI (14.37) recorded highest node for this trait. 
Table.1 Estimation of variability, heritability and genetic advance as per cent of mean for seventeen characters in 45 genotypes of sponge gourd

\begin{tabular}{|c|c|c|c|c|c|c|c|c|}
\hline \multirow[t]{2}{*}{ Sl.No } & \multirow[t]{2}{*}{ Character } & \multicolumn{2}{|c|}{ Range } & \multirow[t]{2}{*}{ Mean } & \multirow[t]{2}{*}{ GCV \% } & \multirow[t]{2}{*}{ PCV \% } & \multirow{2}{*}{$\begin{array}{c}h^{2}(\text { Broad } \\
\text { sense })\end{array}$} & \multirow{2}{*}{$\begin{array}{c}\text { Genetic Adv. As } \\
\% \text { of Mean }\end{array}$} \\
\hline & & Maximum & Minimum & & & & & \\
\hline 1 & $\begin{array}{l}\text { Vine length (m) (90 } \\
\text { DAS) }\end{array}$ & 4.85 & 01.98 & 03.29 & 12.55 & 14.30 & 89.55 & 22.70 \\
\hline 2 & No. of primary branches & 4.80 & 01.80 & 03.09 & 24.56 & 24.94 & 96.99 & 49.83 \\
\hline 3 & $\begin{array}{l}\text { No. of fruiting nodes on } \\
\text { main stem }\end{array}$ & 7.20 & 02.20 & 04.20 & 34.46 & 34.67 & 98.79 & 70.55 \\
\hline 4 & $\begin{array}{l}\text { Days to first staminate } \\
\text { flower appearance }\end{array}$ & 49.85 & 30.88 & 42.26 & 10.71 & 12.45 & 73.91 & 18.96 \\
\hline 5 & $\begin{array}{l}\text { Days to first pistillate } \\
\text { flower appearance }\end{array}$ & 55.50 & 35.23 & 46.54 & 11.13 & 11.89 & 87.58 & 21.45 \\
\hline 6 & Days to $50 \%$ flowering & 60.00 & 37.00 & 50.26 & 12.01 & 13.01 & 85.29 & 22.85 \\
\hline 7 & Span of flowering & 42.23 & 35.27 & 37.63 & 03.71 & 04.85 & 58.69 & 05.86 \\
\hline 8 & Sex ratio $(M / F)$ & 37.15 & 14.93 & 22.76 & 22.29 & 24.35 & 83.81 & 42.04 \\
\hline 9 & $\begin{array}{l}\text { Node of } 1^{\text {st }} \text { female flower } \\
\text { appearance }\end{array}$ & 19.05 & 06.56 & 13.03 & 18.73 & 19.59 & 91.44 & 36.89 \\
\hline 10 & $\begin{array}{l}\text { Days to harvestable } \\
\text { maturity from anthesis }\end{array}$ & 07.45 & 05.30 & 06.45 & 06.95 & 07.54 & 85.07 & 13.21 \\
\hline 11 & Fruit length $(\mathrm{cm})$ & 25.54 & 11.37 & 19.49 & 18.92 & 20.91 & 81.86 & 35.26 \\
\hline 12 & Fruit diameter $(\mathrm{cm})$ & 06.40 & 04.20 & 05.21 & 11.35 & 12.45 & 83.06 & 21.31 \\
\hline 13 & fruit weight (g) & 184.29 & 74.96 & 137.25 & 14.20 & 16.49 & 74.15 & 25.19 \\
\hline 14 & Fruits per plant & 18.89 & 07.41 & 10.93 & 22.97 & 23.61 & 94.58 & 46.01 \\
\hline 15 & No. of seeds per fruit & 125.32 & 47.22 & 90.99 & 16.31 & 20.64 & 62.41 & 26.54 \\
\hline 16 & $\begin{array}{l}\text { Seed index (100 seed } \\
\text { weight) }\end{array}$ & 13.01 & 10.73 & 11.91 & 04.69 & 05.20 & 81.40 & 08.72 \\
\hline 17 & Yield per plant (kg) & 02.26 & 00.65 & 01.46 & 44.16 & 46.66 & 89.55 & 86.08 \\
\hline
\end{tabular}


Table.2 Genotypic (G) and Phenotypic (P) correlation coefficients among important biometrical traits in sponge gourd

\begin{tabular}{|c|c|c|c|c|c|c|c|c|c|c|c|c|c|c|c|c|c|}
\hline 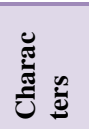 & & (PB) & $(\mathbf{F N})$ & (DSF) & (DFF) & (DPF) & (SF) & (SR) & (NFA) & (DHM) & $(\mathrm{FL})$ & (FD) & (FW) & (FPP) & (NSF) & (SI) & $(\mathbf{Y})$ \\
\hline \multirow[t]{2}{*}{ VL } & G & $0.2705^{* *}$ & -0.0354 & 0.0107 & 0.0425 & 0.1267 & $0.4624 * *$ & $0.4869 * *$ & 0.1294 & -0.0082 & -0.0088 & 0.0309 & 0.0100 & -0.1299 & 0.1251 & $0.5255^{* *}$ & -0.0152 \\
\hline & $\mathrm{P}$ & $0.2696 * *$ & 0.0117 & $0.2494 * *$ & $0.1963^{*}$ & $0.2595^{* *}$ & $0.5590 * *$ & $0.5476^{* * *}$ & $0.2379 * *$ & 0.1481 & 0.1609 & $0.1934 *$ & $0.2200^{*}$ & -0.0184 & $0.3522^{* *}$ & 0.1652 & 0.0353 \\
\hline \multirow[t]{2}{*}{ PB } & G & & $0.8599 * *$ & $-0.6522 * *$ & $-0.6120 * *$ & -0.6030 *** & 0.0667 & $-0.4180 * *$ & $-0.6577 * *$ & $-0.2779 * *$ & 0.1484 & $-0.2539 * *$ & -0.0427 & $0.5040 * *$ & -0.0049 & 0.0353 & $0.4689 * *$ \\
\hline & $\mathrm{P}$ & & 0.8589 ** & $-0.5024 * *$ & $-0.5201 * *$ & $-0.5241 * *$ & 0.1310 & $-0.3537 * *$ & $-0.5992 * *$ & $-0.2089 *$ & $0.1811^{*}$ & $-0.1896^{*}$ & 0.0107 & $0.5149 * *$ & 0.0494 & 0.0101 & 0.4747 ** \\
\hline \multirow[t]{2}{*}{ FN } & G & & & $-0.6712 * *$ & $-0.6665^{* *}$ & $-0.6492 * *$ & -0.1565 & $-0.4872 * *$ & $-0.6833^{* *}$ & $-0.4126^{* *}$ & $0.2340 * *$ & $-0.2589^{* *}$ & 0.1500 & $0.5008^{* *}$ & $0.1877^{*}$ & 0.0408 & 0.6141 ** \\
\hline & $\mathrm{P}$ & & & $-0.5404 * *$ & $-0.5872 * *$ & $-0.5824 * *$ & -0.0689 & $-0.4272 * *$ & $-0.6350^{* *}$ & $-0.3529 * *$ & $0.2442 * *$ & $-0.2078^{*}$ & 0.1603 & $0.5051 * *$ & 0.1837 & 0.0235 & $0.6168^{* *}$ \\
\hline \multirow[t]{2}{*}{ DSF } & $\mathrm{G}$ & & & & $0.9458 * *$ & $0.9543 * *$ & 0.0770 & $0.6166 * *$ & $0.6890 * *$ & $0.4038 * *$ & $-0.5178 * *$ & $0.4299 * *$ & $-0.2273 * *$ & $-0.3273 * *$ & $-0.2245^{* *}$ & 0.0617 & $-0.4906^{* *}$ \\
\hline & $\mathrm{P}$ & & & & $0.9385^{* *}$ & $0.9434 * *$ & $0.2980 * *$ & $0.6340 * *$ & $0.6848^{* *}$ & $0.4706^{* *}$ & $-0.2473 * *$ & $0.5245^{* *}$ & 0.0018 & $-0.1952 *$ & 0.0330 & -0.0773 & $-0.3729 * *$ \\
\hline \multirow[t]{2}{*}{ DFF } & $\mathrm{G}$ & & & & & $0.9530 * *$ & $0.2843 * *$ & $0.5934 * *$ & $0.6781^{* *}$ & $0.5352 * *$ & $-0.5309 * *$ & $0.4759 * *$ & $-0.2119^{* *}$ & $-0.2974 * *$ & $-0.1928^{*}$ & 0.0141 & $-0.4766^{* *}$ \\
\hline & $\mathrm{P}$ & & & & & $0.9512 * *$ & $0.3967 * *$ & $0.6067 * *$ & $0.6818^{* *}$ & $0.5691 * *$ & $-0.3246^{* *}$ & $0.5340^{* *}$ & -0.0467 & $-0.2089 *$ & -0.0015 & -0.0752 & $-0.4024 * *$ \\
\hline \multirow[t]{2}{*}{ DPF } & G & & & & & & $0.2480 * *$ & $0.6529 * *$ & $0.6818^{* *}$ & $0.4750 * *$ & $-0.5302 * *$ & $0.4799 * *$ & $-0.1703 *$ & $-0.3696^{* *}$ & -0.1485 & 0.0476 & $-0.5084 * *$ \\
\hline & $\mathrm{P}$ & & & & & & $0.3429 * *$ & $0.6678 * *$ & $0.6908 * *$ & $0.5132 * *$ & $-0.3493 * *$ & $0.5399 * *$ & -0.0225 & $-0.2857 * *$ & 0.0212 & -0.0464 & $-0.4423^{* *}$ \\
\hline \multirow[t]{2}{*}{ SF } & G & & & & & & & $0.4424 * *$ & 0.1591 & $0.4064 * *$ & $-0.2267 * *$ & 0.1405 & 0.0174 & $-0.1811^{*}$ & 0.0738 & $0.2101^{*}$ & -0.0729 \\
\hline & $\mathrm{P}$ & & & & & & & $0.4604 * *$ & $0.2290 * *$ & $0.4806^{* *}$ & 0.0407 & $0.2815^{* *}$ & $0.2146^{*}$ & -0.0267 & $0.3030 * *$ & -0.0038 & 0.0046 \\
\hline \multirow[t]{2}{*}{ SR } & G & & & & & & & & $0.7187 * *$ & $0.3363 * *$ & -0.1623 & $0.2493 * *$ & $0.1853^{*}$ & $-0.4152 * *$ & $0.2205^{*}$ & $0.2949 * *$ & $-0.2238^{* *}$ \\
\hline & $\mathrm{P}$ & & & & & & & & $0.7295 * *$ & $0.3745^{* *}$ & -0.0488 & $0.3153 * *$ & $0.2759 * *$ & $-0.3306^{* *}$ & $0.3485 * *$ & 0.1002 & $-0.1795^{*}$ \\
\hline \multirow[t]{2}{*}{ NFA } & $\mathrm{G}$ & & & & & & & & & $0.4510^{* *}$ & $-0.2172 *$ & $0.3334 * *$ & -0.0582 & $-0.4297 * *$ & -0.0302 & 0.0585 & $-0.4577 * *$ \\
\hline & $\mathrm{P}$ & & & & & & & & & 0.4639 & -0.1222 & $0.3719^{* *}$ & 0.0510 & $-0.3678 * *$ & 0.0958 & -0.0459 & $-0.4146^{* *}$ \\
\hline \multirow[t]{2}{*}{ DHM } & G & & & & & & & & & & $-0.5552^{* *}$ & $0.3949 * *$ & $-0.2843^{* *}$ & $-0.2328 * *$ & $-0.2520^{* *}$ & 0.0346 & $-0.4148^{* *}$ \\
\hline & $\mathrm{P}$ & & & & & & & & & & $-0.3703 * *$ & $0.4511 * *$ & -0.1199 & -0.1445 & -0.0434 & -0.0540 & $-0.3463 * *$ \\
\hline \multirow[t]{2}{*}{ FL } & G & & & & & & & & & & & $-0.8009^{* *}$ & $0.3530 * *$ & -0.0773 & $0.4026^{* *}$ & $0.3937 * *$ & $0.3309^{* *}$ \\
\hline & $\mathrm{P}$ & & & & & & & & & & & $-0.5656^{* *}$ & $0.4261 * *$ & 0.0052 & $0.4642 * *$ & $0.2280 * *$ & 0.3396 ** \\
\hline \multirow[t]{2}{*}{ FD } & G & & & & & & & & & & & & $-0.4016^{* *}$ & 0.1207 & $-0.3681 * *$ & $-0.2037^{*}$ & $-0.2147^{*}$ \\
\hline & $\mathrm{P}$ & & & & & & & & & & & & $-0.2070 *$ & $0.1711^{*}$ & -0.1344 & $-0.2569 * *$ & -0.1582 \\
\hline \multirow[t]{2}{*}{ FW } & $\mathrm{G}$ & & & & & & & & & & & & & $-0.3733^{* *}$ & $0.9570 * *$ & $0.5530 * *$ & $0.4677^{* * *}$ \\
\hline & $\mathrm{P}$ & & & & & & & & & & & & & $-0.2393 * *$ & $0.9251 * *$ & $0.2974 * *$ & $0.4475 * *$ \\
\hline \multirow[t]{2}{*}{ FPP } & G & & & & & & & & & & & & & & $-0.2855^{* *}$ & -0.1478 & $0.6607^{* * *}$ \\
\hline & $\mathrm{P}$ & & & & & & & & & & & & & & -0.1371 & -0.1649 & $0.6648^{* *}$ \\
\hline \multirow[t]{2}{*}{ NSF } & $\mathrm{G}$ & & & & & & & & & & & & & & & $0.6836^{* *}$ & 0.5401 ** \\
\hline & $\mathrm{P}$ & & & & & & & & & & & & & & & $0.2816^{* *}$ & $0.4772 * *$ \\
\hline \multirow[t]{2}{*}{ SI } & $\mathrm{G}$ & & & & & & & & & & & & & & & & $0.2462 * *$ \\
\hline & $\mathrm{P}$ & & & & & & & & & & & & & & & & $0.1978^{*}$ \\
\hline
\end{tabular}

*: Significant at $\mathrm{p}=0.05$,

**: Significant at $\mathrm{p}=0.01$

(VL)- Vine length (cm); (PB)-No. of primary branches; (FN)-No. of fruiting nodes on main stem; (DSF)-Days to first staminate flower appearance; (DFF)-Days to 50\% flowering; (DPF)-Days to first pistillate flower appearance; (SF)-Span of flowering; (SR)-Sex ratio (M/F); (NFA)-Node at which $1^{\text {st }}$ female flower appearance; (DHM)-Days to harvestable maturity from anthesis; (FL)-Fruit length (cm); (FD)-Fruit diameter (cm); (FW)-Fruit weight (g); (FPP)-Fruits per plant; (NSF)-No. of seeds per fruit; (SI)-Seed index (100 seed weight); (Y)- Yield per plant (kg) 
Table.3 Path Coefficients (Genotypic) showing direct (Bold) and indirect effects of component traits in sponge gourd genotypes

\begin{tabular}{|c|c|c|c|c|c|c|c|c|c|c|c|c|c|c|c|c|c|}
\hline 选 & $(\mathrm{VL})$ & (PB) & $(\mathbf{F N})$ & (DSF) & (DFF) & (DPF) & (SF) & (SR) & (NFA) & (DHM) & (FL) & (FD) & $(\mathbf{F W})$ & (FPP) & (NSF) & (SI) & $\mathbf{r}$ \\
\hline VL & -0.3629 & 0.0461 & 0.0058 & -0.0079 & 0.0163 & -0.0009 & -0.0423 & 0.2268 & -0.0087 & 0.0023 & 0.0029 & -0.0054 & -0.0023 & -0.0911 & 0.0855 & 0.1205 & -0.0152 \\
\hline PB & -0.0982 & 0.1705 & -0.1398 & 0.4825 & -0.2349 & 0.0041 & -0.0061 & -0.1947 & 0.0441 & 0.078 & -0.0488 & 0.0443 & 0.0097 & 0.3535 & -0.0033 & 0.0081 & $0.4689 * *$ \\
\hline FN & 0.0129 & 0.1466 & -0.1626 & 0.4966 & -0.2558 & 0.0044 & 0.0143 & -0.2269 & 0.0458 & 0.1159 & -0.077 & 0.0452 & -0.0339 & 0.3512 & 0.1282 & 0.0094 & $0.6141 * *$ \\
\hline DSF & -0.0039 & -0.1112 & 0.1091 & -0.7398 & 0.363 & -0.0065 & -0.007 & 0.2872 & -0.0462 & -0.1134 & 0.1705 & -0.0751 & 0.0515 & -0.2295 & -0.1534 & 0.0141 & $-0.4906 * *$ \\
\hline DFF & -0.0154 & -0.1043 & 0.1084 & -0.6997 & 0.3838 & -0.0065 & -0.026 & 0.2764 & -0.0455 & -0.1503 & 0.1748 & -0.0831 & 0.048 & -0.2086 & -0.1318 & 0.0032 & $-0.4766 * *$ \\
\hline DPF & -0.046 & -0.1028 & 0.1056 & -0.706 & 0.3657 & -0.0068 & -0.0227 & 0.3041 & -0.0457 & -0.1334 & 0.1745 & -0.0838 & 0.0386 & -0.2592 & -0.1015 & 0.0109 & $-0.5084 * *$ \\
\hline SF & -0.1678 & 0.0114 & 0.0255 & -0.057 & 0.1091 & -0.0017 & -0.0914 & 0.2061 & -0.0107 & -0.1141 & 0.0746 & -0.0245 & -0.0039 & -0.127 & 0.0504 & 0.0482 & -0.0729 \\
\hline SR & -0.1767 & -0.0713 & 0.0792 & -0.4562 & 0.2277 & -0.0044 & -0.0404 & 0.4657 & -0.0482 & -0.0944 & 0.0534 & -0.0435 & -0.0419 & -0.2912 & 0.1507 & 0.0676 & $-0.2238 * *$ \\
\hline NFA & -0.047 & -0.1121 & 0.1111 & -0.5097 & 0.2603 & -0.0046 & -0.0145 & 0.3347 & -0.0671 & -0.1266 & 0.0715 & -0.0582 & 0.0132 & -0.3013 & -0.0207 & 0.0134 & $-0.4577 * *$ \\
\hline DHM & 0.003 & -0.0474 & 0.0671 & -0.2987 & 0.2054 & -0.0032 & -0.0371 & 0.1566 & -0.0303 & -0.2808 & 0.1828 & -0.069 & 0.0643 & -0.1633 & -0.1722 & 0.0079 & $-0.4148 * *$ \\
\hline FL & 0.0032 & 0.0253 & -0.0381 & 0.3831 & -0.2038 & 0.0036 & 0.0207 & -0.0756 & 0.0146 & 0.1559 & -0.3292 & 0.1399 & -0.0799 & -0.0542 & 0.2751 & 0.0903 & $0.3309 * *$ \\
\hline FD & -0.0112 & -0.0433 & 0.0421 & -0.318 & 0.1827 & -0.0032 & -0.0128 & 0.1161 & -0.0224 & -0.1109 & 0.2637 & -0.1747 & 0.0909 & 0.0847 & -0.2515 & -0.0467 & $-0.2147 *$ \\
\hline FW & -0.0036 & -0.0073 & -0.0244 & 0.1682 & -0.0813 & 0.0012 & -0.0016 & 0.0863 & 0.0039 & 0.0798 & -0.1162 & 0.0701 & -0.2264 & -0.2618 & 0.6539 & 0.1268 & $0.4677 * *$ \\
\hline FPP & 0.0471 & 0.0859 & -0.0814 & 0.2421 & -0.1141 & 0.0025 & 0.0166 & -0.1934 & 0.0288 & 0.0654 & 0.0254 & -0.0211 & 0.0845 & 0.7013 & -0.1951 & -0.0339 & $0.6607 * *$ \\
\hline NSF & -0.0454 & -0.0008 & -0.0305 & 0.1661 & -0.074 & 0.001 & -0.0067 & 0.1027 & 0.002 & 0.0708 & -0.1326 & 0.0643 & -0.2166 & -0.2002 & 0.6833 & 0.1567 & $0.5401 * *$ \\
\hline SI & -0.1907 & 0.006 & -0.0066 & -0.0456 & 0.0054 & -0.0003 & -0.0192 & 0.1373 & -0.0039 & -0.0097 & -0.1296 & 0.0356 & -0.1252 & -0.1036 & 0.4671 & 0.2293 & $0.2462 * *$ \\
\hline
\end{tabular}

(VL)- Vine length (m); (PB)-No. of primary branches; (FN)-No. of fruiting nodes on main stem; (DSF)-Days to first staminate flower appearance; (DFF)-Days to 50\% flowering; (DPF)-Days to first pistillate flower appearance; (SF)-Span of flowering; (SR)-Sex ratio (M/F); (NFA)-Node at which ${ }^{\text {st }}$ female flower appearance; (DHM)-Days to harvestable maturity from anthesis; (FL)-Fruit length (cm); (FD)-Fruit diameter (cm); (FW)-Fruit weight (g); (FPP)-Fruits per plant; (NSF)-No. of seeds per fruit; (SI)-Seed index (100 seed weight) 
Table.4 Grouping of 45 sponge gourd genotypes based on D2 analysis

\begin{tabular}{|c|c|c|c|}
\hline S.No & $\begin{array}{c}\text { Cluster } \\
\text { number }\end{array}$ & $\begin{array}{c}\text { No of } \\
\text { genotypes }\end{array}$ & Name of genotypes \\
\hline $\mathbf{1}$ & Cluster-I & 18 & $\begin{array}{c}\text { Basantpur Local, Habra Local, IC-398694, IC-398572, IC-336981, IC-361081, IC-343029, IC-326772, VRSG-57, IC-276519, } \\
\text { Rajahmundry Local, IC-544806, IC-355633, Kalyani Local-2, IC-538115, IC-538688, IC-397534 and Kalyani Local-1 }\end{array}$ \\
\hline $\mathbf{2}$ & Cluster-II & 12 & $\begin{array}{c}\text { IC-336760, IC-284795, IC-092761, IC-336759, IC-549807, IC-276381, VRSG-167, VRSG-70, IC-550776, IC-274593, VRSG-08 and IC- } \\
\text { 284882 }\end{array}$ \\
\hline $\mathbf{3}$ & Cluster-III & 9 & IC-284877, VRSG-09, IC-284869, VRSG-199, IC-276284, IC-339218, Ghoragacha Local, IC-355635 and Kalyani Local-3 \\
\hline $\mathbf{4}$ & Cluster-IV & 3 & IC-343160, VRSG-12 and Patna Local \\
\hline $\mathbf{5}$ & Cluster-V & 1 & IC-284948 \\
\hline $\mathbf{6}$ & Cluster-VI & 1 & IC-284941 \\
\hline $\mathbf{7}$ & Cluster-VII & 1 & IC-284840 \\
\hline
\end{tabular}

Table.5 Inter and intra cluster D2 values

\begin{tabular}{|c|c|c|c|c|c|c|c|}
\hline & Cluster-I & Cluster-II & Cluster-III & Cluster-IV & Cluster-V & Cluster-VI & Cluster-VII \\
\hline Cluster-I & 227.436 & 1643.740 & 555.056 & 2356.755 & 936.269 & 1081.514 & 1568.103 \\
\hline Cluster-II & & 351.528 & 1131.590 & 745.572 & 3399.356 & 2853.304 & 1959.682 \\
\hline Cluster-III & & & 519.616 & 1775.888 & 1552.321 & 1463.971 & 1445.357 \\
\hline Cluster-IV & & & & 541.126 & 5177.897 & 4034.087 & 3552.242 \\
\hline Cluster-V & & & & 0.000 & 966.879 & 1276.876 \\
\hline Cluster-VI & & & & & & 0.000 & 732.866 \\
\hline Cluster-VII & & & & & & & 0.000 \\
\hline
\end{tabular}


Table.6 Mean values of seven clusters for yield and its contributing characters

\begin{tabular}{|c|c|c|c|c|c|c|c|c|c|c|c|c|c|c|c|c|c|}
\hline 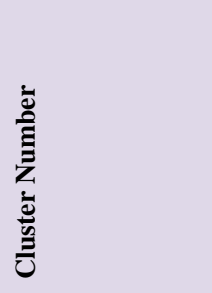 & 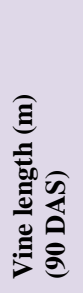 & 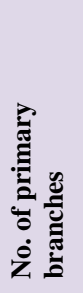 & 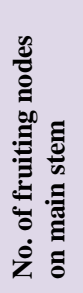 & 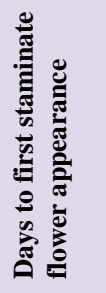 & 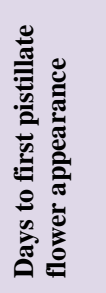 & 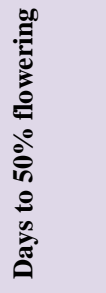 & 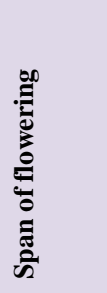 & 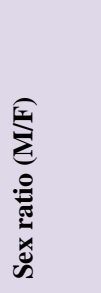 & 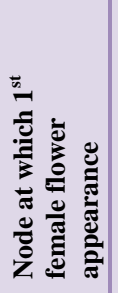 & 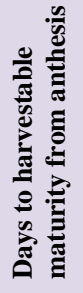 & 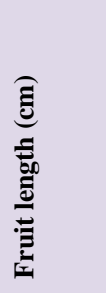 & 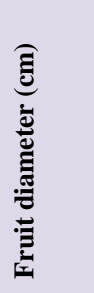 & 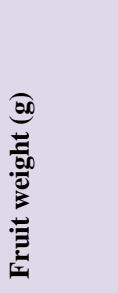 & 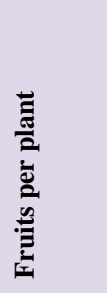 & 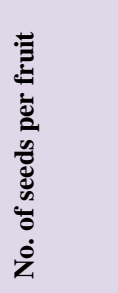 & 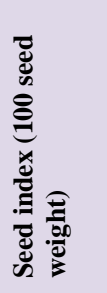 & 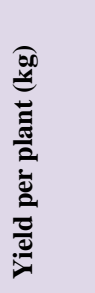 \\
\hline Cluster-I & 3.45 & 2.66 & 3.09 & 43.99 & 48.44 & 52.20 & 37.52 & 24.67 & 14.37 & 6.52 & 19.99 & 5.21 & 136.84 & 09.19 & 89.74 & 11.97 & 1.25 \\
\hline Cluster-II & 3.25 & 3.89 & 5.92 & 39.15 & 42.60 & 45.58 & 37.44 & 20.78 & 11.18 & 6.23 & 20.87 & 4.99 & 145.95 & 12.46 & 96.89 & 11.82 & 1.80 \\
\hline Cluster-III & 3.13 & 2.78 & 3.93 & 43.47 & 48.60 & 53.03 & 38.43 & 23.29 & 13.72 & 6.63 & 18.17 & 5.33 & 137.89 & 10.32 & 91.99 & 12.04 & 1.42 \\
\hline Cluster-IV & 3.25 & 3.13 & 4.87 & 37.95 & 41.71 & 45.08 & 37.30 & 21.06 & 10.72 & 6.02 & 21.43 & 4.95 & 159.75 & 13.41 & 112.22 & 12.62 & 2.14 \\
\hline Cluster-V & 3.22 & 2.80 & 3.20 & 41.00 & 46.83 & 48.50 & 37.40 & 21.10 & 12.83 & 6.84 & 16.29 & 5.12 & 87.28 & 07.49 & 54.99 & 10.73 & 0.65 \\
\hline Cluster-VI & 3.14 & 3.60 & 3.40 & 46.30 & 49.23 & 56.63 & 37.25 & 16.09 & 13.23 & 6.88 & 11.37 & 6.35 & 74.96 & 18.89 & 47.22 & 10.73 & 1.42 \\
\hline Cluster-VII & 2.74 & 3.60 & 5.60 & 47.96 & 52.63 & 57.50 & 36.49 & 20.93 & 12.02 & 6.47 & 11.41 & 6.40 & 79.13 & 17.27 & 49.85 & 10.98 & 1.37 \\
\hline $\begin{array}{l}\% \\
\text { Contribution } \\
\text { towards } \\
\text { divergence }\end{array}$ & 4.20 & 5.31 & 7.27 & 7.84 & 3.76 & 3.48 & 5.18 & 7.31 & 3.15 & 5.14 & 6.89 & 3.54 & 9.48 & 5.78 & 3.43 & 2.91 & 15.33 \\
\hline
\end{tabular}


Cluster- IV (6.02) recorded least number of days to harvestable maturity from anthesis, while cluster- VI (6.88) recorded maximum mean value. Maximum mean for fruit length was in the cluster-IV $(21.43 \mathrm{~cm})$ and minimum for the cluster-VI $(11.37 \mathrm{~cm})$. Diameter of fruit was maximum in the cluster-VII $(6.40 \mathrm{~cm})$ and minimum in the cluster- IV $(4.95 \mathrm{~cm})$. Fruit weight ranged from 74.96 to $159.75 \mathrm{~g}$, with maximum fruit weight in the cluster- IV and minimum in the cluster- VI. Cluster- V (7.49) had recorded minimum number of fruits per plant, while cluster- VI (18.89) maximum number of fruits per plant. Number of seeds per fruit ranged from 47.22 to 112.22 with maximum number of seed per fruit in cluster- IV and minimum in cluster- VI. Seed index was highest in cluster- IV (12.62 g) and least in cluster- V and VI (10.73 g). Yield per plant was highest in the cluster- IV $(2.14 \mathrm{Kg})$ while lowest yield in the cluster $\mathrm{V}(0.65 \mathrm{~kg})$. For crop improvement in sponge gourd inter crossing among genotypes with outstanding mean performance was suggested by Singh et al., (2008) in ridge gourd, Quamruzzaman et al., (2011), Singh et al., (2017) in sponge gourd.

Highest per cent contribution towards total genetic divergence (Table 6) was exhibited by yield per plant (15.33), followed by fruit weight (9.48), Days to first staminate flower appearance(7.84), sex ratio (7.31), number of fruiting nodes on main stem (7.27), fruit length (6.89), fruits per plant (5.78) and Number of primary branches (5.31) together contributing 57.37 percent towards divergence. Hence, these characters should be given high emphasis during hybridization programme. Rest of the characters exhibited $42.63 \%$ contributed towards divergence.

On the basis of this study, it can be concluded that selection would be rewarding for number of primary branches, number of fruiting nodes on main stem, sex ratio, fruits per plant and yield per plant in bringing out the improvement in the sponge gourd as they appeared with high value of GCV, PCV, heritability and genetic gain. Further, correlation study also suggested that for improvement in yield, selection for such plants having more primary branches, fruiting nodes on main stem, fruit length, fruit weight, fruits per plant, seed per fruit and 100 seed weight would be beneficial. Yield per plant and fruit weight were found to be the important characters for increasing the yield potential in sponge gourd.

\section{References}

Ananthan and Krishnamoorthy. 2017. Genetic Variability, Correlation and Path analysis in ridge gourd (Luffa acutangula (Roxb) L.). Int. J. Curr. Microbiol. App. Sci., 6(6): 3022-3026.

Choudhary, B.R., Sudhakar Pandey., Singh, P.K and Pandey, V. 2014. Genetic diversity analysis for quantitative traits in hermaphrodite ridge gourd [Luffa acuangulata (Roxb.) L.]. Indian J. Hort., 71(2): 284-287.

Choudhary, B.R., Sudhakar Pandey., Singh, P.K., Pandey, V and Ramesh Singh. 2011. Genetic divergence in hermaphrodite ridge gourd (Luffa acutangula). Vegetable Science, 38(1): 68-72.

Dubey, R.K., Vikas, S and Upadhyay, G. 2013. Variability, Interrelationship and Path Analysis for yield improvement in Luffa. International Journal of Vegetable Science, 19:342-351.

Gopalan, C., Sastri, V., Balasubramanium, S. C., Rao, B.S.N., Dosthale, Y.G and Pant, K. C. 1999. Nutritive value of Indian foods. Indian Council of Medical Research Technological Bulletin. National Institute of Nutrition, Hyderabad. Pp. 51.

Gowda, H. K., Shirol, A. M., Mulge, R., 
Shantappa, T and Prasadkumar. 2011. Genetic variability, heritability and genetic advance for yield and yield contributing characters in ridge gourd (Luffa acutangula (L.) Roxb), Journal of Asian Horticulture, 7 (4): 196-200.

Johnson, H. W., Robinson, H. F and Comstock, R. E. 1955. Estimates of genetic and environmental variability in soybean. Agronomy Journal, 47: 314318.

Kalloo, G., 1993. Loofah-Luffa spp. In: Genetic Improvement of Vegetable Crops. G. Kalloo. and B. O. Bergh. (Eds). Pergamon Press., pp 265-266

Karthik, D., Varalakshmi, B., Kumar, G and Lakshmipathi, N. 2017. Genetic variability studies of ridge gourd advanced inbred lines (Luffaacutangula (L.) Roxb.). Int. J. Pure App. Biosci., 5(6): 1223-1228.

Khule, A. A., Tikka, S. B. S., Jadhav, D. J and Kajale, D. B. 2011. Genetic variability and heritability studies in local collections of sponge gourd (Luffa cylindrical Roem). Asian Journal of Bio Science, 6(1):119-120.

Khule, A. A., Tikka, S. B. S., Jadhav, D. J and Kajale, D. B. 2012. Genetic divergence in local collections of sponge gourd (Luffa cylindrica (L.) M. Roem.). Indian Journal of Tropical Biodiversity. 20(1): 77-81.

Koppad, S. B., Chavan, M. L., Hallur, R. H., Rathod, V and Shantappa, T. 2015. Variability and character association studies in ridge gourd (Luffa acutangula L.) with reference to yield attributes. Journal of Global Biosciences, 4(5): 2332-2342.

Kumar, R., Ameta K. D., Dubey, R. B and Pareek, S. 2013. Genetic variability, correlation and path analysis in sponge gourd (Luffa cylindrical Roem.). African Journal of Biotechnology, 12(6):539-543.
Lush, J. L., 1949. Heritability of quantitative characters in farm animals. Proc. of $85^{\text {th }}$ Congress Genetics Heredities, Supplement, pp 356-375.

Mahalanobis, P. C., 1936. On generalized distance in statistics. Proceedings of national institute of science. 2: 49-55.

More, T. A and Shinde, K. G. 2001. Ridge and Sponge gourd. In: Vegetables, tuber crops and spices. S. Thamburaj. and Singh. N. (Eds). Indian Council of Agricultural Research.p 309.

Narasannavar, A. R., Gasti, V. D., Shantappa, T., Mulge, R., Allolli, T. B and Thammaiah, N. 2014.Heterosis studies in ridge gourd [Luffa acutangula (L.) Roxb.]. Karnataka J. Agric. Sci., 27(1): 47-51.

Pandey, V., Singh, V. B and Singh, Achala. 2012. Correlation and Path Analysis of yield and yield contributing traits for higher yield and better marketable traits in sponge gourd (Luffa cylindrical roem.). Progressive Horticulture, 44(1): 126-129.

Panse, V. G and Sukhatme, P. V. 1985.Statistical methods for agricultural workers. $2^{\text {nd }}$ Ed. ICAR, New Delhi.

Quamruzzaman, A. K. M., Ahmad, S., Moniruzzaman, M., Chowdhury, M. A. $\mathrm{Z}$ and Mollah, M. A. H. 2011. Genetic diversity analysis of sponge gourd (Luffa cylindrical Roem.) in Bangladesh. SAARC J. Agri., 9(2):4551.

Rabbani, M.G., Naher, M.J and Hoque, S. 2012. Variability, Character association and Diversity analysis of ridge gourd (Luffa acutangula Roxb.) genotypes of Bangladesh. SAARC J. Agri., 10(2): 110.

Robinson, H. F., 1966. Quantitative genetics in relative to breeding and impact of mendalism in agriculture, biology and medicine. Indian Journal of Genetics, 26:171-187. 
Sharma, N., Bisen, B. P., Rajani, B and Verma, B. 2017. Genetic variability, correlation and path analysis in sponge gourd genotypes in Kymore plateau. Green Farming, 8(2): 301-305.

Singh, R. D and Singh, J. P. (2009). Sponge gourd. In: Hand book of horticulture. Chada, K. L. (Eds). ICAR, New Delhi, p 463.

Singh, B. D., (2000). Plant BreedingPrinciples and Methods (6th Edition). Kalyani Publishers, New Delhi, India. Pp. 896.

Singh, D. K., Maurya, S. K., Jaiswal, H. R. and Singh, A. 2008. Studies on genetic variability and genetic divergence analysis in ridge gourd. Progressive Horticulture. 40(2):149-154.

Singh, D. K., Maurya, S. K., Jaiswal, H. R., Singh, $\mathrm{A}$ and Lohani, $\mathrm{M}$. 2012.Character association and path coefficient analysis in ridge gourd (Luffa acutangula (L.)) Roxb. Pantnagar Journal of Research, 10(2): 189-195.

Swarup, V., 2006. Cucurbits, In: Vegetable Science and Technology in India.
Kalyani Publishers, Kolkata. Pp. 426.

Takahashi, H., 1980. Sex expression as effected by N6 benzylaminopurine in staminate inflorescence of Luffa cylindrica. Plant Cell Physiol., 21: 525536.

Varalakshmi, B., Pitchaimuthu, M., Sreenivas, R. E., Manjunath, K. S and Swathi, S. H. 2015. Genetic Variability, Correlation and Path Analysis in ridge gourd [Luffa acutangula (Roxb.) L.]. Journal of Horticultural Sciences, 10(2): 154-158.

Yadav, A. N., Singh, V. B., Yadav, G. C and Kumar, V. 2017. Determining relationships between different horticultural and yield traits in sponge gourd (Luffa cylindrical Roem.) genotypes with path coefficient analysis. Journal of Pharmacognosy and Phytochemistry, 6(3): 342-345

Yadav, H., Maurya, S. K and Bhatt, L. 2016. Genetic divergence in ridge gourd through principal component and nonhierarchical cluster analysis. Journal Environment and Ecology, 34 (1): 334340.

\section{How to cite this article:}

Suresh Kumar, J., M.K. Pandit and Lakshmi Pathy, T. 2019. Genetic Variability, Diversity and Character Association in Sponge Gourd [Luffacylindrica (Roem.) L.]. Int.J.Curr.Microbiol.App.Sci. 8(03): 278-290. doi: https://doi.org/10.20546/ijcmas.2019.803.035 